\title{
An Adaptive Distance-Based Location Update Algorithm for Next-Generation PCS Networks
}

\author{
Vincent W. S. Wong, Member, IEEE, and Victor C. M. Leung, Senior Member, IEEE
}

\begin{abstract}
In this paper, we propose a stochastic model to compute the optimal update boundary for the distance-based location update algorithm. The proposed model is flexible and captures some of the real characteristics in the wireless cellular environment. The model can adapt to arbitrary cell topologies in which the number of neighboring base stations at different locations may vary. The cell residence time can follow general distributions which captures the fact that the mobile user may spend more time at certain locations than others. The model also incorporates the concept of a trip in which the mobile user may follow a particular path to a destination. For implementation, the decision of location update can be made by a simple table lookup. Numerical results indicate that the proposed model provides a more accurate update boundary in real environment than that derived from a hexagonal cell configuration with a random walk movement pattern. The proposed model allows the network to maintain a better balance between the processing incurred due to location update and the radio bandwidth utilized for paging between call arrivals.
\end{abstract}

Index Terms-Location update, PCS networks.

\section{INTRODUCTION}

I $\mathrm{N}$ RECENT years, there has been a significant increase in the number of personal communications service (PCS) subscribers around the world. The next-generation PCS networks will provide other new services (e.g., Internet access) besides voice. In order to utilize the radio spectrum efficiently, a cellular architecture is used in wireless networks. The geographical coverage area is partitioned into cells, each served by a base station. Mobile users and their terminals ${ }^{1}$ are connected to the network via the base stations. Cells can have different sizes: picocells are commonly used in indoor environments; microcells are used within city centers; and macrocells are used to cover highways and suburban and rural areas. Smaller cells use less power for transmissions and allow a greater frequency reuse, but require more network overhead for mobility management.

One of the issues in mobility management is to track the locations of the users. Since mobile users are free to move within the

Manuscript received December 30, 2000; revised June 27, 2001. This work was supported in part by the Natural Sciences and Engineering Research Council of Canada under a Postgraduate Scholarship and Grant OGP0044286 and scholarships from the Communications Research Centre, Industry Canada, and the University of British Columbia. This work was presented in part at IEEE ICC 2001, Helsinki, Finland, June 2001.

The authors are with the Department of Electrical and Computer Engineering, University of British Columbia, Vancouver, BC V6T 1Z4, Canada (e-mail: vincentw@ece.ubc.ca; vleung@ece.ubc.ca).

Publisher Item Identifier S 0733-8716(01)08481-5.

${ }^{1}$ In the remainder of this paper, we use the terms "mobile" user and "mobile terminal" interchangeably. coverage area, the network can only maintain the approximate location of each user. When a connection needs to be established to a particular user, the network has to determine the user's exact location within the cell granularity. The operation of the mobile terminal informing the network about its current location is known as location update, and the operation of the network determining the exact location of the mobile user for the purpose of call notification is called terminal paging or searching.

It is well known that there is a tradeoff between location update and paging. If the mobile terminal updates its location whenever it crosses a cell boundary, then the network can maintain its precise location. However, if the call arrival rate is low, the network wastes its resources by processing frequent update information and the mobile terminal wastes its power by transmitting the update signal. On the other hand, if the mobile terminal does not perform a location update frequently, a large coverage area has to be paged when a call arrives, which wastes the radio bandwidth. Thus, the central problem of location management is to devise algorithms which minimize the overall cost for location update and paging.

We now describe some related work on location update. Interested readers can also refer to [1] or [2] for a comprehensive survey on location management for various wireless networks. Location update algorithms can be divided into two main groups: static and dynamic. In a static algorithm, location update is triggered based on the topology of the network. Examples include the conventional location area (LA)-based scheme used in GSM and IS-41 systems. In a dynamic algorithm, location update is based on the user's call and mobility patterns. Examples include the distance-based, timer-based, and the movement-based schemes. In the distance-based scheme [3]-[5], a mobile terminal transmits an update signal whenever its distance (in terms of the number of cells) from the previous update location exceeds a certain threshold. In the timer-based scheme [3], [6], a mobile terminal updates its location periodically. In the movement-based scheme [3], [7], [8], a location update is performed when the number of cell boundary crossings from the previous update location exceeds a certain value. Numerical results in [3] show that the distance-based update scheme has a better performance in terms of a lower overall cost for location update and paging when compared to the timer-based and movement-based schemes.

A number of novel location update algorithms have also been proposed recently. In an adaptive threshold scheme [9], a mobile terminal transmits an update message every $T^{\prime}$ time units, where the parameter $T^{\prime}$ is not a constant but varies with the current signaling load on the uplink control channel of the cell. In the predictive distance-based update scheme [10], the mobile terminal 
reports both its location and velocity during the update process. Based on this information, the network predicts the mobile terminal's location in future time. The mobile terminal checks its position periodically and performs a location update whenever its distance exceeds the threshold distance measured from the predicted location. In [11], a state-based update scheme is analyzed where the system state includes the current location and the time elapsed since the last update. The LeZi update algorithm proposed in [12] can be considered to be a path-based update scheme in which the movement history rather than the current location is sent in an update message. The movement history consists of a list of IDs of zones (LAs or cells) the mobile terminal has crossed after the last update. Last but not least, the concepts of boundary location area and boundary location register were proposed in [13] to facilitate roaming between different wireless networks. The update distance is a function of the QoS factor, velocity ratio, and predefined threshold.

Our work focuses on the determination of the optimal update boundary for the distance-based location update algorithm. Although it has been shown that the distance-based update algorithm has a better performance than the LA, movement, and timer based update schemes, the models used to determine the optimal distance threshold are often based on certain simplifying assumptions. These assumptions are also commonly used in other location update algorithms. First of all, structured cell configurations are commonly used. For example, mesh or hexagonal cell configurations are used in two-dimensional models (e.g., [5], [7], and [13]), and a linear model is used in the one-dimensional case (e.g., [3], [4], and [10]). Although these cell topologies simplify the analytical computation, they do not give an accurate representation of a realistic cellular network topology, where the size of each cell depends on the transmit power, receiver sensitivity, antenna radiation pattern, and propagation environment, and the number of neighboring cells varies from cell to cell. We believe a graph model such as the one proposed in [14] is more appropriate to characterize the topology of a cellular network. In a graph model, each node can represent a base station. An edge between two nodes represents that the two base stations are neighbors.

Another commonly used assumption is related to the cell residence time (also known as the cell dwell time) distribution. The cell residence time denotes the amount of time that the mobile terminal stays in a particular cell before moving to another one. Most of the work assumed the cell residence time follows a geometric (or exponential) distribution (e.g., [3], [4], and [10]) that is independent and identically distributed (i.i.d.) for all the cells in the network. The major limitation of the i.i.d. geometric residence time assumption is that it does not capture an accurate representation of the mobility pattern of each individual user, who may stay at certain locations (e.g., the user's home or office) for a relatively long period of time. To the best of our knowledge, only the model proposed in [7] allows an i.i.d. general distribution for all cells, and the model proposed in [14] allows different exponentially distributed cell residence times at different cells.

The last assumption is related to the movement models. The symmetric random walk is commonly used to characterize individual movement behavior (e.g., [3]-[5], [7], [9], and [13]). In this model, when a mobile user leaves a cell, there is an equal probability that the user will move to any one of the neighboring cells. Although the random walk model simplifies the analysis, its main drawback is that the direction of the mobile user is not taken into account. In general, a mobile user usually travels with a specific destination in mind. Thus, the mobile's location in the future is likely to be correlated with its movement history.

In this paper, we propose a new model to determine the optimal update boundary for the distance-based location update algorithm in a realistic wireless environment. We formulate the location tracking problem as a semi-Markov decision process [15]. There is a cost function associated with location update and another cost function associated with terminal paging. The objective is to determine the optimal update boundary so as to minimize the expected total cost between call arrivals. Distinct features of our model include [16], [17] the following.

1) Applicable to arbitrary cell topologies: This feature captures the fact that the number of neighboring base stations at different locations may vary in real life. Some base stations may only have two neighboring base stations while others can have as many as six. Thus, our model is not restricted to structured cell configurations such as mesh or hexagonal. The graph model will be explained in detail in Section IV.

2) Cell residence time can follow general distributions: This captures the fact that the mobile user may spend more time at certain cell locations (e.g., home or office) than some other locations. In addition, various distributions can be used to model different cell sizes (e.g., macrocell, microcell, or picocell). The average residence time in each cell can be different. The i.i.d. exponential or geometric cell residence time assumption can be relaxed.

3) Incorporate the movement history: The probability that the mobile user moves to a particular neighboring cell can depend on the location of the current cell or a list of cells recently visited. This movement pattern can incorporate the concept of a trip in which the mobile user may follow a particular path to a destination. The assumption of a symmetric random walk movement pattern can be relaxed.

The structure of this paper is as follows. In Section II, we introduce the notations and describe the model formulation. In Section III, we analyze the distance-based algorithm under a given cell residence time distribution and describe its implementation in an arbitrary cell topology. Numerical results are presented in Section IV. Conclusions are given in Section V. A list of notations is shown in Table I.

\section{MODEL FORMULATION}

In this section, we describe how to formulate the location tracking problem as a semi-Markov decision process. The notations that we use follow those described in [15]. A Markov decision process model consists of five elements: decision epochs, states, actions, transition probabilities, and costs. The mobile terminal has to make a decision whenever it crosses a cell boundary or a certain time has elapsed. Those time instants are called decision epochs. Referring to Fig. 1, the sequence $\sigma_{0}, \sigma_{1}, \ldots$ represents the times of successive decision epochs. Since the network must track the user's location perfectly 
TABLE I

LIST OF NOTATIONS

\begin{tabular}{|c|c|}
\hline Notation & Description \\
\hline$\sigma_{n}$ & Time at decision epoch $n$. \\
\hline$T$ & Arrival time of the next call. \\
\hline$A$ & Action set. \\
\hline$Y_{n}$ & Action chosen at decision epoch $n$. \\
\hline$X_{n}$ & State at decision epoch $n$. \\
\hline$f\left(X_{n}, Y_{n}\right)$ & $\begin{array}{l}\text { Location update cost at decision epoch } n \text {, given current state is } X_{n} \text { and action } Y_{n} \\
\text { is chosen. }\end{array}$ \\
\hline$C_{L U}$ & Fixed location update cost. \\
\hline$\phi(T)$ & Last decision epoch before the next call arrival. \\
\hline$h\left(X_{\phi(T)}\right)$ & Cost incurred on terminal paging. \\
\hline$\delta_{t}$ & Action choice when the system occupies state $s$ at decision epoch $t$. \\
\hline$\pi$ & Policy. \\
\hline$v^{\pi}(s)$ & Expected total cost between call arrivals given policy $\pi$ is used with initial state $s$. \\
\hline$G\left(t \mid X_{n}, Y_{n}\right)$ & $\begin{array}{l}\text { Cumulative distribution function of the time between decision epochs } n \text { and } n+1 \text {, } \\
\text { given current state is } X_{n} \text { and action } Y_{n} \text { is chosen. }\end{array}$ \\
\hline$\lambda$ & Call arrival rate. \\
\hline$\mu$ & Cell crossing rate ( $1 / \mu$ corresponds to the average cell residence time). \\
\hline$P[j \mid s, \delta(s)]$ & $\begin{array}{l}\text { Transition probability that the next state is } j \text {, given current state is } s \text { and action } \\
\delta(s) \text { is chosen. }\end{array}$ \\
\hline$C_{P}$ & Paging cost per cell. \\
\hline$\hat{\lambda}$ & Estimated call arrival rate. \\
\hline$\Delta_{\lambda}$ & Percentage change of the average time between call arrivals. \\
\hline$X_{0}, Y_{0}$ & $X_{\phi(T)}, Y_{\phi(T)}$ \\
\hline$\sigma_{0}=0$ & $\operatorname{\sigma orrr}_{1} \tau_{2} \rightarrow \sigma_{2} \quad \cdots \quad \sigma_{\phi(T)} \quad T$ \\
\hline
\end{tabular}

Fig. 1. Timing diagram.

during a call, the user's location is known to the network when a call terminates. Thus, the time interval requiring mobility tracking is between the termination of the last call and the arrival of the next one. In Fig. $1, \sigma_{0}=0$ denotes the last call termination time and the random variable $T$ represents the arrival time of the next call.

At each decision epoch, the mobile terminal has to decide whether to update its location or not. The action set $A=\{0,1\}$, where " 1 " represents the action of performing location update and " 0 " represents the null action of no intervention. The random variable $Y_{n}$ is used to denote the action chosen at decision epoch $n$.

The mobile terminal chooses an action based on its current state information. The state information can include: the number of cell boundary crossings since the last update (movementbased), the cell distance between the current location and where the previous update was performed (distance-based), the velocity of the mobile terminal, or some other criteria. The random variable $X_{n}$ is used to denote the state at decision epoch $n$.

Two cost functions are introduced to account for the network resources used for location update and terminal paging. The location update cost reflects the consumption of radio bandwidth and battery power, as well as the update processing incurred on the mobile terminal location database. The paging cost reflects the number of cells being paged and the number of search iterations performed before the mobile terminal is located.

The function $f\left(X_{n}, Y_{n}\right)$ denotes the location update cost at decision epoch $n$, given that the current state is $X_{n}$ and action 
$Y_{n}$ is chosen. The location update cost is assumed to be fixed and is denoted by $C_{L U}$. Thus, $f\left(X_{n}, 1\right)=C_{L U}$ and $f\left(X_{n}, 0\right)=0$.

For the paging cost function, referring to Fig. 1, let $\phi(T)$ denote the last decision epoch before the next call arrival. The cost function $h\left(X_{\phi(T)}\right)$ represents the cost incurred on terminal paging. We assume that the paging strategy follows the shortest distance-first order [5]. That is, when a paging event occurs, the search is conducted first at the user's last reported cell. If the mobile is not found there, then the search is conducted in increasing distance order from the last reported cell, until the user is located. The maximum paging delay corresponds to the maximum number of search iterations allowed. At each search iteration, a set of cells is paged simultaneously. This paging strategy has also been used in conjunction with other location update algorithms (e.g., [4], [5], and [10]).

A decision rule prescribes a procedure for action selection in each state at a specified decision epoch. Deterministic Markovian decision rules are functions $\delta_{t}: S \rightarrow A$, which specify the action choice when the system occupies state $s$ at decision epoch $t<T$. A policy $\pi=\left(\delta_{1}, \delta_{2}, \ldots\right)$ is a sequence of decision rules to be used at all decision epochs.

Since the time interval requiring mobility tracking is between the termination of the last call and the arrival of the next one, the total cost for location update and paging is calculated within this time interval. Let $v^{\pi}(s)$ denote the expected total cost between call arrivals given policy $\pi$ is used with initial state $s$. It is the sum of the location update and paging costs. Based on the above notations

$$
v^{\pi}(s)=E_{s}^{\pi}\left\{\sum_{n=0}^{\phi(T)} f\left(X_{n}, Y_{n}\right)+h\left(X_{\phi(T)}\right)\right\}
$$

where $E_{s}^{\pi}$ denotes the expectation with respect to policy $\pi$ and initial state $s$. In the above expression, the first term corresponds to the lump sum portion of the location update cost. The second term corresponds to the paging cost incurred upon a call arrival.

Let $G\left(t \mid X_{n}, Y_{n}\right)$ denote the cumulative distribution function of the time between decision epochs $n$ and $n+1$, given current state $X_{n}$ and chosen action $Y_{n}$. The time between decision epochs corresponds to the cell residence time. In our formulation, the cell residence time follows a general distribution that can depend on the location of the cell. This captures the fact that a mobile user may spend more time at certain cell locations than the others. In addition, different cell sizes (e.g., macrocell, microcell, picocell) can yield different cell residence time distributions. Thus, the usual i.i.d. exponential cell residence time assumption can be relaxed.

If the time between call arrivals at each mobile terminal is exponentially distributed with mean $1 / \lambda$, then as shown in the Appendix, (1) can be written as

$$
v^{\pi}(s)=E_{s}^{\pi}\left\{\sum_{n=0}^{\infty} e^{-\lambda \sigma_{n}} c\left(X_{n}, Y_{n}\right)\right\}
$$

where

$$
\begin{aligned}
c\left(X_{n}, Y_{n}\right)= & f\left(X_{n}, Y_{n}\right) \\
& +h\left(X_{n}\right) \int_{0}^{\infty}\left(1-e^{-\lambda \tau}\right) G\left(d \tau \mid X_{n}, Y_{n}\right)
\end{aligned}
$$

The cost function $c\left(X_{n}, Y_{n}\right)$ can be interpreted as the effective cost incurred at decision epoch $n$, given that the current state is $X_{n}$ and action $Y_{n}$ is chosen.

A policy is said to be stationary if $\delta_{t}=\delta$ for all $t$. A stationary policy has the form $\pi=(\delta, \delta, \ldots)$; for convenience we denote it by $\delta$. For a stationary policy $\delta,(2)$ can be written as

$$
\begin{aligned}
v^{\delta}(s)= & c[s, \delta(s)] \\
& +\sum_{s^{\prime} \in S}\left\{\int_{0}^{\infty} e^{-\lambda t} v^{\delta}\left(s^{\prime}\right) P\left[s^{\prime} \mid s, \delta(s)\right] G[d t \mid s, \delta(s)]\right\}
\end{aligned}
$$

where $P\left[s^{\prime} \mid s, \delta(s)\right]$ denotes the transition probability that the next state is $s^{\prime}$, given that the current state is $s$ and action $\delta(s)$ is chosen. For a proof of this fact, please see the Appendix. Our objective is to determine an optimal stationary deterministic policy $\delta^{*}$ which minimizes (4). Note that for Markovian movement patterns, correlations between the directions of successive moves of a mobile user can also be incorporated if the state includes a history of the cells visited.

\section{Distance-Based Location Update Algorithm}

In this section, we begin by describing how the model formulated in Section II can be used to analyze the distance-based location update algorithm. The optimality equations are introduced. We then discuss the implementation issues based on a table lookup. Extension of the model to include the movement history is also described.

\section{A. Optimality Equations}

For the distance-based location update algorithm, we let the state $s=(i, j)$ where $i$ represents the identifier of the cell in which the mobile terminal is currently residing, and $j$ represents the identifier of the cell in which the mobile terminal performed its last update. Based on the notations introduced in Section II, the paging cost function is given by $h(i, j)$. For a particular mobile user, different cell residence time distributions can be assigned to different cell locations. The average residence time in each cell can be different. This is achieved by letting $G(t \mid i)$ denote the cumulative distribution function of the cell residence time, given the identifier of the current cell is $i$. This captures the fact that a mobile user may spend more time at certain cell locations than the others. In this paper, we use $G(d t \mid i)$ to represent the time-differential. That is, $G(d t \mid i)=d G(t \mid i)$.

For the movement pattern in an arbitrary cell topology, we let $P(k \mid i)$ denote the probability that the mobile user will move to neighboring cell $k$ when it leaves cell $i$. This captures the correlations of the user movement between two neighboring cells. Correlations between the directions of successive moves of the mobile user can also be incorporated if the state includes a history of the cells visited. This will be described in Section III-D. Note that the above formulation does not assume any particular cell configurations. Therefore, the proposed model can adapt to arbitrary cell topologies.

Let $v(i, j)$ denote the minimum expected total cost between call arrivals given state $(i, j)$. That is

$$
v(i, j)=\min _{\pi \in \Pi} v^{\pi}(i, j)
$$


The optimality equations are given by

$$
\begin{aligned}
v(i, j)=\min \{ & \int_{0}^{\infty}\left(1-e^{-\lambda t}\right) h(i, j) G(d t \mid i) \\
& +\sum_{k}\left[\int_{0}^{\infty} e^{-\lambda t} v(k, j) P(k \mid i) G(d t \mid i)\right] \\
& C_{L U}+\int_{0}^{\infty}\left(1-e^{-\lambda t}\right) h(i, i) G(d t \mid i) \\
& \left.+\sum_{k}\left[\int_{0}^{\infty} e^{-\lambda t} v(k, i) P(k \mid i) G(d t \mid i)\right]\right\}
\end{aligned}
$$

where $1 \leq i, j \leq N$ and $N$ denotes the number of base stations within the coverage area. The first term in (6) denotes the expected total cost if no update is performed at state $(i, j)$, while the second term denotes the expected total cost if location update is performed at state $(i, j)$.

\section{B. Value Iteration Algorithm}

There are a number of iteration algorithms available to solve (6). Examples include the value iteration, policy iteration, and linear programming algorithms [15]. Value iteration is the most widely used and best understood algorithm for solving Markov decision systems. The following value iteration algorithm finds a stationary deterministic optimal policy and the corresponding expected total cost. The solutions of the optimality equations correspond to the minimum expected total cost $v(i, j)$ and the optimal policy $\delta^{*}(i, j)$. Note that the optimal policy $\delta^{*}(i, j)$ indicates the decision whether to update the mobile terminal's location or not at state $(i, j)$.

Algorithm:

1) Set $v^{0}(i, j)=0$ for each state $(i, j)$ where $1 \leq i, j \leq$ $N$. Specify $\varepsilon>0$ and set $n=0$.

2) For each state $(i, j)$ where $1 \leq i, j \leq N$, compute $v^{n}(i, j)$ by

$$
\begin{aligned}
& v^{n+1}(i, j) \\
& =\min \left\{\int_{0}^{\infty}\left(1-e^{-\lambda t}\right) h(i, j) G(d t \mid i)\right. \\
& +\sum_{k}\left[\int_{0}^{\infty} e^{-\lambda t} v^{n}(k, j) P(k \mid i) G(d t \mid i)\right], \\
& C_{L U}+\int_{0}^{\infty}\left(1-e^{-\lambda t}\right) h(i, i) G(d t \mid i) \\
& \left.+\sum_{k}\left[\int_{0}^{\infty} e^{-\lambda t} v^{n}(k, i) P(k \mid i) G(d t \mid i)\right]\right\}
\end{aligned}
$$

3) If $\left\|v^{n+1}-v^{n}\right\|<\varepsilon$, go to Step 4). Otherwise increment $n$ by 1 and return to Step 2).
4) For each state $(i, j)$ where $1 \leq i, j \leq N$, the stationary optimal policy $\delta^{*}(i, j)=0$ if

$$
\begin{aligned}
& \int_{0}^{\infty}\left(1-e^{-\lambda t}\right) h(i, j) G(d t \mid i) \\
&+\sum_{k}\left[\int_{0}^{\infty} e^{-\lambda t} v^{n}(k, j) P(k \mid i) G(d t \mid i)\right] \\
&<C_{L U}+\int_{0}^{\infty}\left(1-e^{-\lambda t}\right) h(i, i) G(d t \mid i) \\
& \quad+\sum_{k}\left[\int_{0}^{\infty} e^{-\lambda t} v^{n}(k, i) P(k \mid i) G(d t \mid i)\right] .
\end{aligned}
$$

Otherwise, $\delta^{*}(i, j)=1$.

5) Stop.

There are a number of definitions for the function norm $\|$.$\| .$ In this paper, the function norm is defined as $\|v\|=\max v(i, j)$ for $1 \leq i, j \leq N$. Convergence of the value iteration algorithm is ensured since the operation in Step 2) corresponds to a contraction mapping. Thus, the function $v^{n}(i, j)$ converges in norm to $v(i, j)$. Note that the convergence rate of the value iteration algorithm is linear.

Based on the optimal policy $\delta^{*}(i, j)$, the optimal update boundary can be determined. Since the mobile terminals usually have limited processing power, the computations can be performed at either the base stations or some designated switches.

\section{Implementation Considerations}

Having identified the different parameters involved in the model, we are now in a position to explain the steps that need to be taken in order to implement the model. First of all, the network controller has to assign cost functions for location update and terminal paging. It also has to maintain the mobility profile of each mobile user (i.e., its movement history and call history). Based on this information, the average residence time in each cell as well as the average call arrival rate can be estimated [18].

Given the input parameters (i.e., cost functions and various distributions), the value iteration algorithm can be used to determine the optimal update boundary for each mobile terminal. The optimal update boundary is then stored in a database (e.g., location register). After each location update or a call termination, the mobile terminal needs to download the list of the update boundary cell identifiers that corresponds to its current location. Whenever the mobile terminal moves to another cell, it compares the new cell identifier with the list of the update boundary cell identifiers. A location update is performed if the new cell is one of those update boundary cells.

The optimal update boundary stored in the location register needs to be revised whenever there is a change of the movement history, call history, or network topology (e.g., installation of new base stations). The calculation of the optimal update boundaries can be performed offline, e.g., whenever spare processing capacity is available at the network controller. 


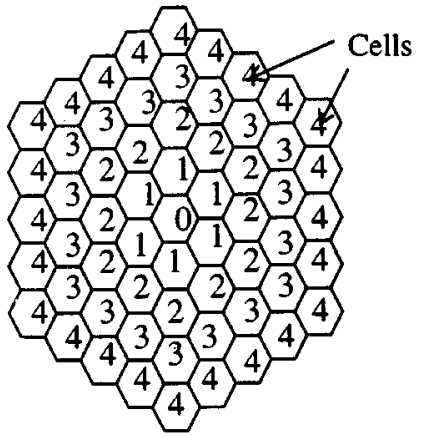

(a)

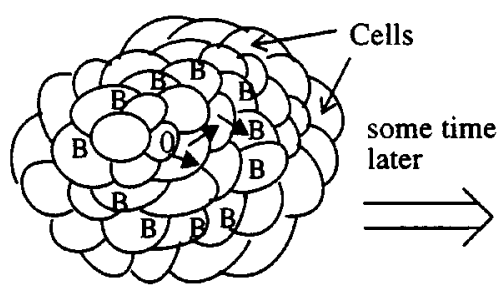

$\longrightarrow$ Mobile's movement

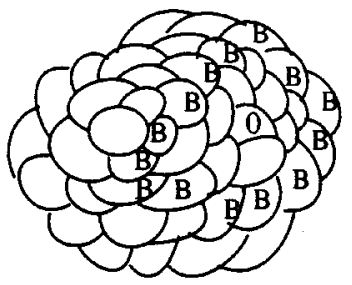

Cell "B": Boundary cell

(b)

Fig. 2. (a) Distance threshold derived from a hexagonal cell configuration. Location update is performed whenever the mobile terminal moves to any cells labeled with a "4". (b) Update boundary derived from an arbitrary cell configuration. Location update is performed whenever the mobile terminal moves to any cells labeled with a "B."

\section{Model Extension to Incorporate Movement History}

The model can incorporate the concept of a trip in which the mobile user may follow a particular path to a specific destination. The model formulation of the distance-based update scheme with movement history is similar to that described in Section III-B; only the state space needs to be extended. In this formulation, we let $s=\left(i_{1}, i_{2}, \ldots, i_{m}, j\right)$ where $\left(i_{1}, i_{2}, \ldots, i_{m}\right)$ represents the list of identifiers of the cells that the mobile terminal has recently visited, with $i_{1}$ representing the most recent one (i.e., the cell in which the mobile terminal is currently residing), and $j$ represents the identifier of the cell in which the mobile terminal performed its last update. The movement correlation is characterized by the probability function $P\left(k \mid i_{1}, i_{2}, \ldots, i_{m}\right)$ which denotes the probability that the mobile user will move to neighboring cell $k$ when it leaves cell $i_{1}$, given that it has visited cells $\left(i_{1}, i_{2}, \ldots, i_{m}\right)$ recently. The cost functions as well as the cell residence distribution remain unchanged. The optimality equations are as follows:

$$
\begin{aligned}
& v\left(i_{1}, i_{2}, \ldots, i_{m}, j\right)\left\{\int_{0}^{\infty}\left(1-e^{-\lambda t}\right) h\left(i_{1}, j\right) G\left(d t \mid i_{1}\right)\right. \\
&=\min \left\{\sum _ { k } \left[\int_{0}^{\infty} e^{-\lambda t} v\left(k, i_{1}, \ldots, i_{m-1}, j\right)\right.\right. \\
&\left.\cdot P\left(k \mid i_{1}, i_{2}, \ldots, i_{m}\right) G\left(d t \mid i_{1}\right)\right] \\
& C_{L U}+ \int_{0}^{\infty}\left(1-e^{-\lambda t}\right) h\left(i_{1}, i_{1}\right) G\left(d t \mid i_{1}\right) \\
&+\sum_{k}\left[\int_{0}^{\infty} e^{-\lambda t} v\left(k, i_{1}, \ldots, i_{m-1}, i_{1}\right)\right. \\
&\left.\left.\left.\cdot P\left(k \mid i_{1}, i_{2}, \ldots, i_{m}\right)\right] G\left(d t \mid i_{1}\right)\right]\right\} .
\end{aligned}
$$

The value iteration algorithm described in Section III-B can be used to evaluate the expected total cost and the optimal policy.

In order to estimate the function $P\left(k \mid i_{1}, i_{2}, \ldots, i_{m}\right)$, the network needs to collect the statistics of the mobile's movement. When a new mobile user is added to the network, the network can simply assume a random walk movement pattern for that mobile user and calculate the update boundary. The mobile terminal needs to store a list of cells recently visited. Whenever the mobile terminal performs a location update, it has to transmit the list of the cell identifiers visited between the two location update events. The network uses this information to estimate the movement history and calculate the new update boundary. Note that how the value of $m$ is chosen is beyond the scope of this paper. Interested readers can refer to [12] for further discussion on movement history.

\section{NUMERICAL RESUlTS AND DisCUSSIONS}

In this section, we first explain the difference between the distance thresholds determined from our model and those derived from a hexagonal cell configuration. We then describe the assumptions and specifications used in our simulation study and present the results in terms of 1) performance comparisons between the distance thresholds determined from our model to those derived from a hexagonal cell configuration and 2) a sensitivity analysis of the optimal distance threshold with respect to the variation of the average cell residence time and call arrival rate.

\section{A. Cell Topologies}

We now describe the differences between the distance thresholds computed in an arbitrary cell topology to those derived from a structured cell topology. In a structured cell topology (e.g., hexagonal), the number of neighboring cells is the same for all cell locations. Since the derivation of the optimal distance threshold in a structured cell configuration is under the assumptions of symmetric random walk and i.i.d. cell residence time distribution, the optimal distance threshold is the same for all cell locations. As an example, see Fig. 2(a).

On the other hand, in an arbitrary cell topology, the number of neighboring cells can be different at different cell locations. 


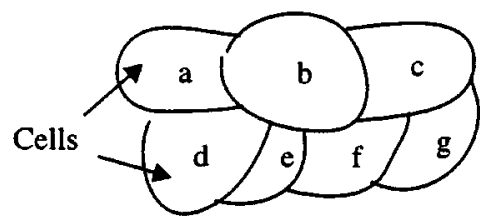

(a)

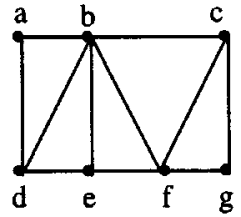

(b)
Fig. 3. Representation of a cellular network topology by a graph model.

Since our proposed model allows Markovian movement pattern and general cell residence time distributions at different cell locations:

1) for any cell located on the update boundary, its cell distance measured from the last update cell location may be different from those of other cells located on the update boundary;

2) different cell locations may have different update boundaries.

As an example, refer to Fig. 2(b). The cell labeled with a " 0 " is the cell where a location update was last performed. The update boundary consists of the set of cells labeled with a "B." Note that some boundary cells have cell distances from cell "0" equal to 2 while some have cell distances equal to 3 . Whenever the mobile terminal moves to any of the boundary cells, a location update is performed. After an update is performed in a boundary cell, that cell will be labeled as "0" and a new set of boundary cells will be chosen.

\section{B. Simulation Assumptions and Specifications}

In our simulation environment, a graph model is used to represent the topology of a cellular network. In general, the adjacency of the cells can be modeled as a connected graph $G=(N, E)$, where the node set $N$ represents the set of cell or base station identifiers and the edge set $E$ represents the neighboring cells. For example, referring to Fig. 3, the node set $N=\{a, b, c, d, e, f, g\}$ and the edge set $E=\{(a, b),(a, d),(b, c), \ldots(f, g)\}$.

In particular, we use a random graph model to represent the topology of a cellular network. The rationale behind using a random graph model is that: 1) the number of neighboring base stations for each base station can be different and 2) only the nodes that are close together are connected. This models the adjacency of the neighboring cell sites. The procedures of generating random graphs can be found in [19] and [20]. In our model, we consider a coverage area that consists of 100 base stations with an average node degree ${ }^{2}$ of 6 . An example of a random graph model is shown in Fig. 4.

Since the mobile user usually has a destination in mind, we model this behavior by choosing one particular node (or cell) in the random graph as the destination. Whenever the mobile user leaves the current cell, it moves to a neighboring cell which is closest to the destination. This captures the behavior of moving toward the destination. If the mobile user is staying within the destination cell, after a certain period of time it will move to

\footnotetext{
${ }^{2}$ The average node degree is defined as the average number of links connected to a node.
}

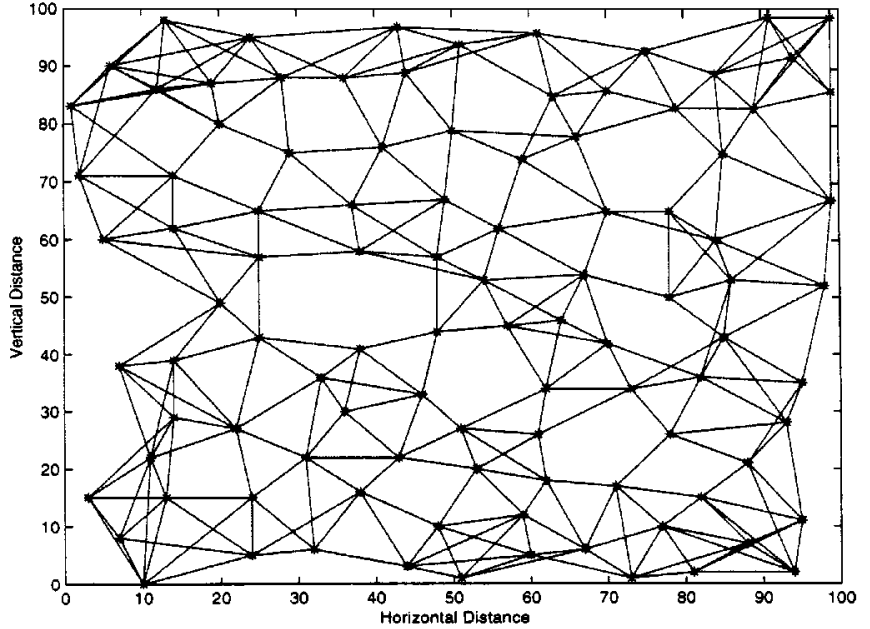

Fig. 4. A random graph model with an average node degree of 6 . The nodes represent the location of the base stations. An edge between two nodes represents those two base stations that are neighbors.

one of the neighboring cells. This continues until the next call arrives.

We now describe the procedures of comparing the distance thresholds determined from our model to those derived from a hexagonal cell configuration. The goal is to show that the proposed model gives a more accurate update boundary in real wireless cellular environments compared with that derived from a hexagonal cell configuration with random walk movement pattern. The steps for the comparison are described as follows.

1) Given the cost and mobility parameters, we first use a hexagonal cell configuration with symmetric random walk movement pattern to obtain the distance threshold.

2) This distance threshold is then applied to the random graph model with Markovian movement pattern. The expected total cost of location update and paging between call arrivals, denoted as "Cost (hexagonal)," is then determined. The term "hexagonal" is used to remind us that the distance threshold is derived from the hexagonal cell configuration.

3) For our proposed model, we also use the random graph model with Markovian movement pattern to determine the minimum expected total cost by solving the optimality equations in (6). This cost is denoted as "Cost (optimal)." The term "optimal" is used to remind us that the update boundary corresponds to the optimal policy.

4) The performance gain is the cost ratio which is defined as Cost (hexagonal)/Cost (optimal).

Unless stated otherwise, the parameters that we use in the following subsections are: call arrival rate $\lambda=0.01$ per minute, the location update cost $C_{L U}=10$, and the paging cost per cell $C_{P}=1$. We assume that the cell residence time follows an i.i.d. Gamma distribution with average time $1 / \mu$. For the value iteration algorithm, we choose $\varepsilon=10^{-10}$.

\section{Results}

Fig. 5 shows the relative frequency distribution of the average optimal distance threshold. For each last updated location, its average optimal distance threshold is defined as the average of 


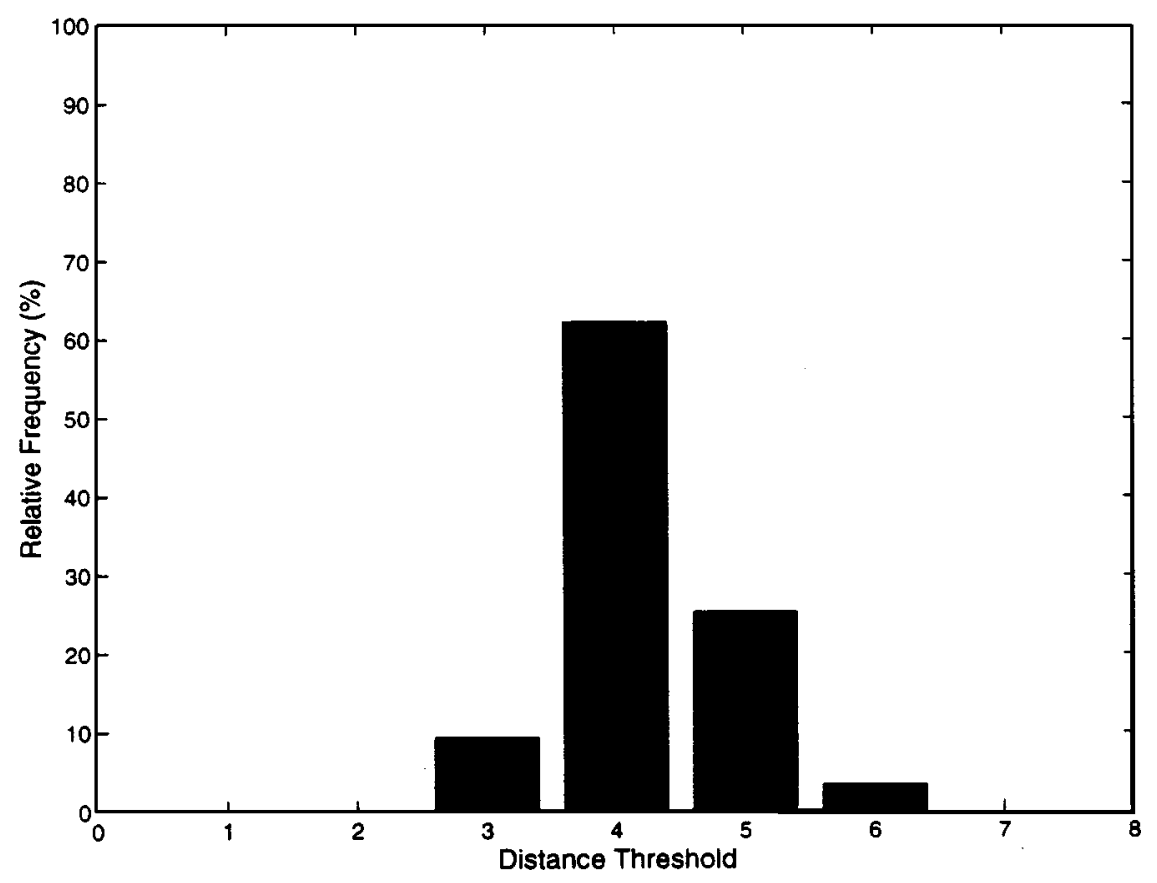

Fig. 5. Optimal distance threshold distribution.

(a)

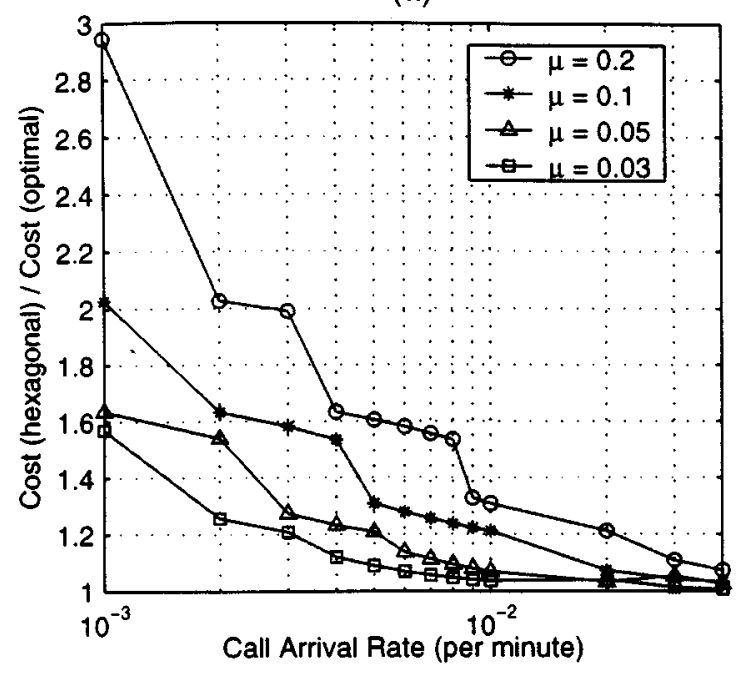

(b)

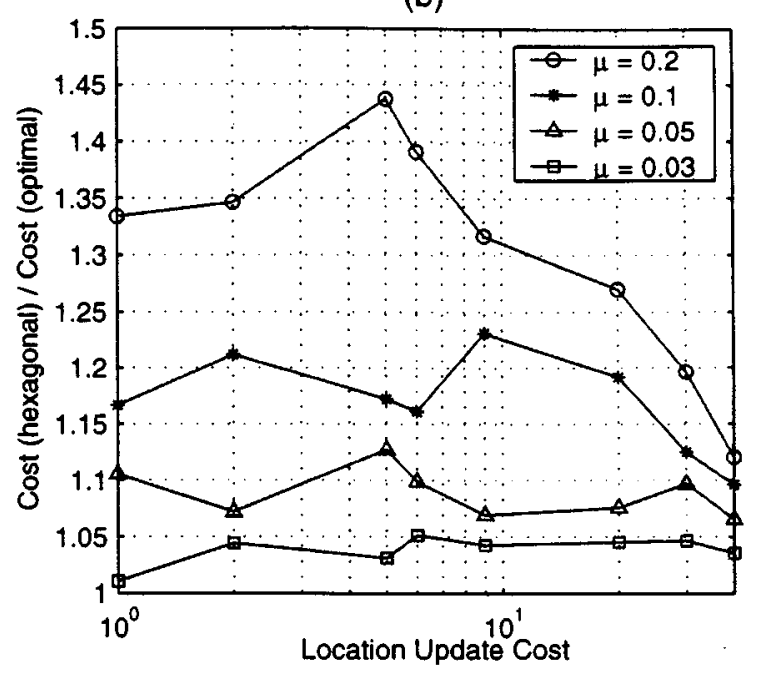

Fig. 6. (a) Cost ratio versus call arrival rate $\lambda$ under different cell crossing rates $\mu\left(C_{L U}=10, C_{P}=1\right)$. (b) Cost ratio versus location update cost $C_{L U}$ under different cell crossing rates $\mu\left(\lambda=0.01, C_{P}=1\right)$.

all the cell distance of the update boundary cells relative to the last updated cell location. For illustration purposes, the optimal distance threshold is rounded to the closest integer. Fig. 5 shows that for a random graph topology, approximately $62 \%$ of the cells have a distance threshold of $4,25 \%$ of the cells have a distance threshold of $5,10 \%$ of the cells have a distance threshold of 3 , and $3 \%$ of the cells have a distance threshold of 6 . Note that, for the same set of cost and mobility parameters, the optimal distance threshold derived from a hexagonal cell configuration is equal to 4 .

Fig. 6(a) and (b) shows the cost ratio versus the call arrival rate $\lambda$ and the location update cost $C_{L U}$ under different cell crossing rates $\mu$ (per minute). ${ }^{3}$ From these two figures, we observe that the cost ratio is always greater than one. That is, Cost (hexagonal) $\geq$ Cost (optimal). This implies that the proposed model provides a more accurate update boundary than that derived from a hexagonal cell configuration with random walk movement pattern.

In Fig. 6(a), when the average time between call arrivals is large (i.e., $\lambda$ is small), the optimal update boundary obtained from our model gives a much lower cost than the distance threshold derived from the hexagonal model. On the other

${ }^{3}$ Note that the inverse (or reciprocal) of the average cell residence time is the cell crossing rate. 
(a)

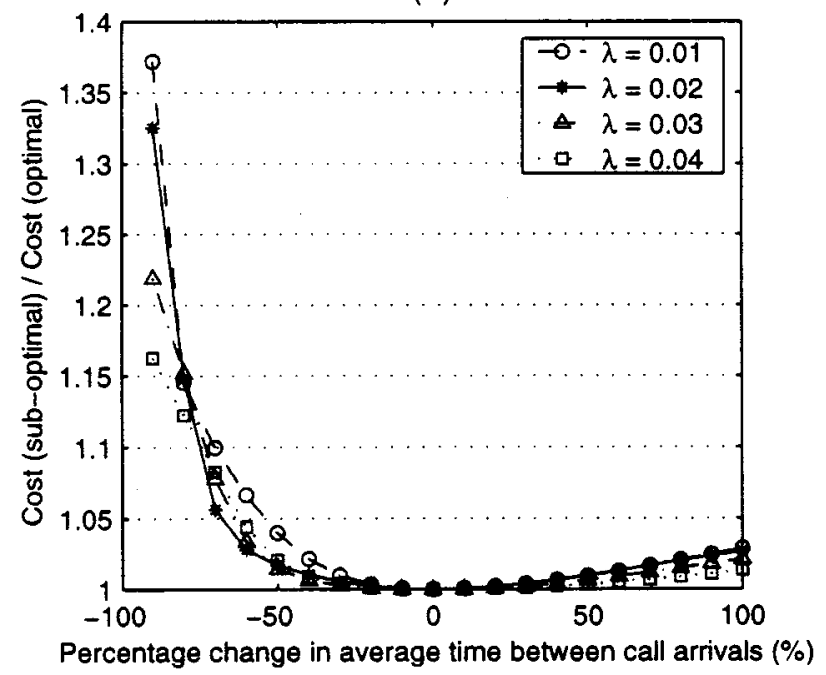

(b)

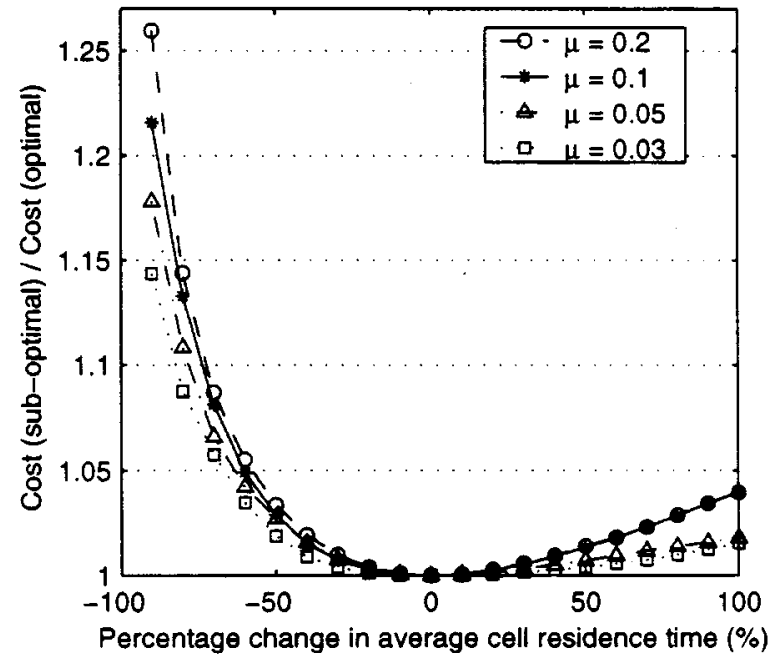

Fig. 7. (a) Cost ratio versus $\Delta_{\lambda}$ under different call arrival rates $\lambda$ ( $\mu=0.1, C_{L U}=10, C_{P}=1$ ). (b) Cost ratio versus $\Delta_{\mu}$ under different cell crossing rates $\mu\left(\lambda=0.01, C_{L U}=10, C_{P}=1\right)$.

hand, when the average time between call arrivals is small (i.e., $\lambda$ is large), the mobile user does not travel much before a call arrives. There is a high chance that the mobile user is staying at the cell where the last location update or call termination was performed. Since we assume that the shortest distance-first paging strategy is used, the expected total cost mainly consists of the paging cost when $\lambda$ is large. In that case, the distance thresholds derive from both methods give the same performance.

In Fig. 6(b), the optimal update boundary obtained from our model gives a lower cost than the distance threshold derived from the hexagonal model. Note that the variation of the cost ratio with respect to $C_{L U}$ is due to the changes of the optimal update boundary or distance threshold for different update cost values. When the location update cost $C_{L U}$ increases, there is less incentive to perform location update. In that case, the expected total cost mainly consists of the paging cost. Since both models used the same paging scheme, we observe that the cost ratio approaches unity as $C_{L U}$ increases.

In real cellular wireless network environments where the cell topology is not structured and the user movement pattern is not random, these results show that our model can provide a more accurate update boundary than that derived from a hexagonal cell configuration with random walk movement pattern. By using a more accurate location update boundary, the network can maintain a better balance between the processing incurred due to location update and the radio bandwidth utilized for paging between call arrivals, thus resulting in a lower overall cost for location update and paging.

In our simulation studies, we found the value iteration algorithm to be efficient and stable. The number of iterations is quite predictable from point to point, changing slowly as the parameter changes. In general, the number of iterations to convergence does not depend on the cost parameters (i.e., $C_{L U}, C_{P}$ ) but depends on the values of $\lambda$ and $\mu$. As an example, for the optimal policy in Fig. 6(b), the value iteration algorithm required only 57 iterations to converge when $\mu=0.03$, but it required 316 iterations when $\mu=0.2$. Note that there are other iteration algorithms available (e.g., policy iteration algorithm) which have a higher rate of convergence. Interested readers can refer to [15] for details.

\section{Sensitivity Analysis}

In order to determine the minimum expected total cost and the update boundary, the optimal policy needs to be evaluated. The optimal policy is a function of $\lambda, \mu$, and other cost parameters. Although the cost parameters can be determined by the network operator, the values of the call arrival rate $\lambda$ and cell crossing rate $\mu$ may not always be estimated correctly. If that is the case, the resulting policy may not indeed be optimal. We are interested in determining the percentage changes of the expected total cost as functions of the variations of the average time between call arrivals and the average cell residence time. The procedures for the sensitivity analysis consist of the following steps.

1) Given the actual call arrival rate $\lambda$ and other cost and mobility parameters, we first determine the minimum expected total cost, denoted as Cost (optimal).

2) Let $\hat{\lambda}$ denote the estimated call arrival rate and $\Delta_{\lambda}$ denote the percentage change of the average time between call arrivals. These parameters are related by the following:

$$
\hat{\lambda}^{-1}=\left(1+\Delta_{\lambda}\right) \lambda^{-1} \text {. }
$$

Based on the estimated call arrival rate $\hat{\lambda}$ and other parameters, the suboptimal policy is determined. From this suboptimal policy and other cost and mobility parameters (i.e., $\lambda$, $\mu$, etc.), the suboptimal expected total cost, denoted as Cost (suboptimal), is computed.

3) The change of the expected total cost with respect to the variation of the average time between call arrivals is characterized by the cost ratio, which is defined as Cost (sub-optimal)/Cost (optimal).

We also use the similar procedures described above to investigate the changes of the expected cost due to the variations of the average cell residence time. Fig. 7(a) shows the cost ratio versus 
$\Delta_{\lambda}$ under different call arrival rates $\lambda$ and Fig. 7(b) shows the cost ratio versus $\Delta_{\mu}$ under different cell crossing rate $\mu$. From these figures, we observe that the cost ratio is more sensitive to the underestimation of both $\lambda^{-1}$ and $\mu^{-1}$. Note that a small increase in the average time between call arrivals corresponds to a small decrease in the call arrival rate. On the other hand, a small decrease in the average time between call arrivals corresponds to a large increase in the call arrival rate. A large difference from the actual parameter gives a larger deviation from the minimum expected total cost. Thus, in Fig. 7(a), we observe that a decrease in the average time between call arrivals gives a higher cost ratio than an increase of the same percentage. The same explanation holds for the average cell residence time case in Fig. 7(b). These results imply that if there is uncertainty in estimating $\lambda^{-1}$ or $\mu^{-1}$, it may be better to overestimate the values in order to reduce the cost ratio difference.

\section{CONCLUSION}

In this paper, we have proposed a stochastic model to analyze the distance-based location update algorithm. The location tracking problem is formulated as a semi-Markov decision process. Based on the current state information, the mobile terminal decides whether to update its location whenever it crosses a cell boundary. The proposed model can adapt to arbitrary cell topologies. The Markovian movement pattern allows the study of a number of mobility models. For example, if the state information includes the identifiers of the recently visited cells, then a history-based mobility model for a particular user can be obtained. The cell residence time follows a general distribution. The usual i.i.d. assumption for the cell residence time distribution can be relaxed.

For the distance-based location update algorithm, we have described its implementation in an arbitrary cell topology. After each location update or a call termination, the mobile terminal needs to download the list of the update boundary cell identifiers corresponding to its current location. Whenever the mobile terminal moves to another cell, it compares the new cell identifier with the list of the update boundary cell identifiers. A location update is performed if the new cell is one of those update boundary cells.

Numerical results indicate that the proposed model gives a more accurate update boundary (or distance threshold) in real wireless cellular environments compared with that derived from a hexagonal cell configuration with random walk movement pattern. By using a more accurate location update boundary, the network can maintain a better balance between the processing incurred due to location update and the radio bandwidth utilized for paging between call arrivals. Results from the sensitivity analysis show that if there is uncertainty in estimating the average time between call arrivals or the average cell residence time, it may be better to overestimate the values in order to reduce the cost ratio difference.

Although the proposed model captures some the characteristics in a real wireless cellular network environment (e.g., arbitrary cell topology, generalized cell residence time per user), the model is not without drawbacks. In our formulation, the call arrival rate for a particular mobile user follows a Poisson distribu- tion. Results in [21] show that it may not be the case. This points to the need for new analytical models for location tracking under general call arrival distributions.

\section{APPENDIX}

In this Appendix, we derive the expression for $v^{\pi}(s)$, the expected total cost between call arrivals given a policy $\pi$ and initial state $s$. The notations used follow those defined in Section II. If the time between call arrivals is exponentially distributed with rate $\lambda$, (1) can be written as

$$
\begin{aligned}
& v^{\pi}(s) \\
& =E_{s}^{\pi}\left\{\int_{0}^{\infty}\left[\sum_{n=0}^{\phi(T)} f\left(X_{n}, Y_{n}\right)+h\left(X_{\phi(T)}\right)\right] \lambda e^{-\lambda t} d t\right\} .
\end{aligned}
$$

If $m$ represents the last decision epoch before the next call arrival, then

$$
\begin{gathered}
v^{\pi}(s)=E_{s}^{\pi}\left\{\sum_{m=0}^{\infty} \int_{\sigma_{m}}^{\sigma_{m+1}}\left[\sum_{n=0}^{m} f\left(X_{n}, Y_{n}\right)+h\left(X_{m}\right)\right]\right. \\
\left.\cdot \lambda e^{-\lambda t} d t\right\} .
\end{gathered}
$$

By interchanging the order of summation of the first term and performing integration for the second term

$$
\begin{aligned}
v^{\pi}(s)=E_{s}^{\pi}\left\{\sum_{n=0}^{\infty} \sum_{m=n}^{\infty} \int_{\sigma_{m}}^{\sigma_{m+1}} f\left(X_{n}, Y_{n}\right) \lambda e^{-\lambda t} d t\right. \\
\left.+\sum_{m=0}^{\infty}\left[e^{-\lambda \sigma_{m}}-e^{-\lambda \sigma_{m+1}}\right] h\left(X_{m}\right)\right\} .
\end{aligned}
$$

Since $\sum_{m=n}^{\infty} \int_{\sigma_{m}}^{\sigma_{m+1}}(\cdot) d t=\int_{\sigma_{n}}^{\infty}(\cdot) d t$

$$
\begin{aligned}
v^{\pi}(s)=E_{s}^{\pi}\left\{\sum_{n=0}^{\infty} \int_{\sigma_{n}}^{\infty} f\left(X_{n}, Y_{n}\right) \lambda e^{-\lambda t} d t\right. \\
\left.+\sum_{m=0}^{\infty}\left[e^{-\lambda \sigma_{m}}-e^{-\lambda \sigma_{m+1}}\right] h\left(X_{m}\right)\right\} .
\end{aligned}
$$

By performing integration for the first term and letting $m=n$ for the second term, we obtain

$$
\begin{aligned}
v^{\pi}(s)=E_{s}^{\pi}\left\{\sum _ { n = 0 } ^ { \infty } \left[e^{-\lambda \sigma_{n}} f\left(X_{n}, Y_{n}\right)\right.\right. \\
\left.\left.+\left(e^{-\lambda \sigma_{n}}-e^{-\lambda \sigma_{n+1}}\right) h\left(X_{n}\right)\right]\right\}
\end{aligned}
$$

which can be simplified to

$$
\begin{aligned}
v^{\pi}(s)=E_{s}^{\pi}\left\{\sum_{n=0}^{\infty} e^{-\lambda \sigma_{n}}\right. \\
\left.\qquad \cdot\left[f\left(X_{n}, Y_{n}\right)+\left(1-e^{-\lambda \tau_{n+1}}\right) h\left(X_{n}\right)\right]\right\}
\end{aligned}
$$

where $\sigma_{n+1}=\sigma_{n}+\tau_{n+1}$. Let $c(s, a)$ denote the expected total cost between two decision epochs, given that the system occupies state $s$ at the first decision epoch and that the deci- 
sion-maker chooses action $a$ in state $s$. If we assume the cost, transition probabilities, and sojourn times are time homogeneous, then

$$
\begin{aligned}
c(s, a) & =f(s, a)+E_{s}^{a}\left\{\left(1-e^{-\lambda \tau_{1}}\right) h(s)\right\} \\
& =f(s, a)+\int_{0}^{\infty}\left(1-e^{-\lambda \tau_{1}}\right) h(s) G\left(d \tau_{1} \mid s, a\right) .
\end{aligned}
$$

Substituting (10) into (9), we obtain

$$
v^{\pi}(s)=E_{s}^{\pi}\left\{\sum_{n=0}^{\infty} e^{-\lambda \sigma_{n}} c\left(X_{n}, Y_{n}\right)\right\} .
$$

For stationary deterministic policy $\delta$

$$
\begin{aligned}
v^{\delta}(s)= & c[s, \delta(s)]+E_{s}^{\delta}\left\{e^{-\lambda \tau_{1}} v^{\delta}\left(X_{1}\right)\right\} \\
= & c[s, \delta(s)] \\
& +\sum_{s^{\prime} \in S}\left\{\int_{0}^{\infty} e^{-\lambda t} v^{\delta}\left(s^{\prime}\right) P\left[s^{\prime} \mid s, \delta(s)\right] G[d t \mid s, \delta(s)]\right\} .
\end{aligned}
$$

\section{ACKNOWLEDGMENT}

The authors would like to thank the anonymous reviewers as well as M. Puterman and M. Lewis for their comments on an earlier draft of this paper.

\section{REFERENCES}

[1] I. F. Akyildiz, J. McNair, J. Ho, H. Uzunalioglu, and W. Wang, "Mobility management in next-generation wireless systems," Proc. IEEE, vol. 87, pp. 1347-1384, Aug. 1999.

[2] V. Wong and V. Leung, "Location management for next generation personal communication networks," IEEE Network, vol. 14, pp. 18-24, Sept./Oct. 2000.

[3] A. Bar-Noy, I. Kessler, and M. Sidi, "Mobile users: To update or not to update?," ACM/Baltzer J. Wireless Networks, vol. 1, no. 2, pp. 175-195, July 1995.

[4] U. Madhow, M. Honig, and K. Steiglitz, "Optimization of wireless resources for personal communications mobility tracking," IEEE/ACM Trans. Networking, vol. 3, pp. 698-707, Dec. 1995.

[5] J. Ho and I. F. Akyildiz, "Mobile user location update and paging under delay constraints," ACM/Baltzer J. Wireless Networks, vol. 1, no. 4, pp. 413-425, Dec. 1995.

[6] C. Rose, "Minimizing the average cost of paging and registration: A timer-based method," ACM/Baltzer J. Wireless Networks, vol. 2, no. 2, pp. 109-116, June 1996.

[7] I. F. Akyildiz, J. Ho, and Y.-B. Lin, "Movement-based location update and selective paging for PCS networks," IEEE/ACM Trans. Networking, vol. 4, pp. 629-638, Aug. 1996.

[8] J. Li, H. Kameda, and K. Li, "Optimal dynamic mobility management for PCS networks," IEEE/ACM Trans. Networking, vol. 8, pp. 319-327, June 2000.

[9] Z. Naor and H. Levy, "Minimizing the wireless cost of tracking mobile users: An adaptive threshold scheme," in Proc. IEEE INFOCOM'98, San Francisco, CA, Mar./Apr. 1998, pp. 720-727.

[10] B. Liang and Z. Haas, "Predictive distance-based mobility management for PCS networks," in Proc. IEEE INFOCOM'99, New York, NY, Mar. 1999.

[11] C. Rose, "State-based paging/registration: A greedy technique," IEEE Trans. Vehic. Technol., vol. 48, pp. 166-173, Jan. 1999.

[12] A. Bhattacharya and S. K. Das, "LeZi-Update: An information-theoretic approach to track mobile users in PCS networks," in Proc. ACM/IEEE MobiCom'99, Seattle, WA, Aug. 1999, pp. 1-12.
[13] W. Wang and I. F. Akyildiz, "Intersystem location update and paging schemes for multitier wireless networks," in Proc. ACM MobiCom'00, Boston, MA, Aug. 2000.

[14] S. K. Sen, A. Bhattacharya, and S. K. Das, "A selective location update strategy for PCS users," ACM/Baltzer J. Wireless Networks, vol. 5, no. 5, pp. 313-326, Sept. 1999.

[15] M. L. Puterman, Markov Decision Processes: Discrete Stochastic Dynamic Programming. New York: Wiley, 1994.

[16] V. Wong, "Stochastic control of inter-switch handoff and location update in wireless cellular networks," Ph.D. dissertation, Univ. British Columbia, Canada, 2000.

[17] V. Wong and V. Leung, "An adaptive distance-based location update algorithm for PCS networks," in Proc. IEEE ICC'01, Helsinki, Finland, June 2001.

[18] M. Zonoozi and P. Dassanayake, "User mobility modeling and characterization of mobility patterns," IEEE J. Select. Areas Commun., vol. 15, pp. $1239-1252$, Sept. 1997.

[19] M. B. Doar, "Better model for generating test networks," in Proc. IEEE GLOBECOM'96, London, U.K., 1996, pp. 86-93.

[20] E. W. Zegura, K. L. Calvert, and M. J. Donahoo, "A quantitative comparison of graph-based models for Internet topology," IEEE/ACM Trans. Networking, vol. 5, pp. 770-783, Dec. 1997.

[21] J. Jannink, D. Lam, N. Shivakumar, J. Widom, and D. Cox, "Efficient and flexible location management techniques for wireless communication systems," ACM/Baltzer J. Wireless Networks, vol. 3, no. 5, pp. 361-374, Oct. 1997.

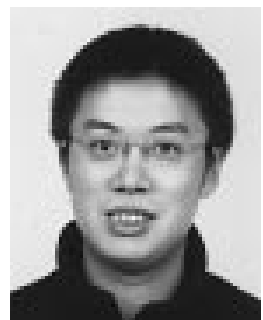

Vincent W. S. Wong (S'93-M'00) received the B.Sc. degree from the University of Manitoba in 1994, the M.A.Sc. degree from the University of Waterloo in 1996, and the Ph.D. degree from the University of British Columbia (UBC) in 2000, all in electrical engineering.

Currently, he is a Systems Engineer at PMC-Sierra, Inc., in Burnaby, BC, Canada. He is also an Adjunct Professor in the Department of Electrical and Computer Engineering at UBC. His research interests include resource allocation and mobility management in wireless networks, IP over DWDM, and protection switching in optical transport networks.

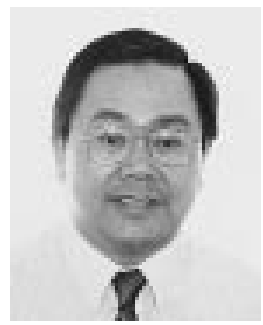

Victor C. M. Leung (S'75-M'79-SM'97) received the B.A.Sc. (Hons.) degree in electrical engineering from the University of British Columbia (UBC) in 1977 and the Ph.D. degree in electrical engineering from UBC on a Natural Sciences and Engineering Research Council Postgraduate Scholarship in 1981.

From 1981 to 1987, he was a Senior Member of Technical Staff at MPR Teltech Ltd., specializing in the planning, design, and analysis of satellite communication systems. He also held a part-time position as Visiting Assistant Professor at Simon Fraser University in 1986 and 1987. In 1988, he was a Lecturer in the Department of Electronics at the Chinese University of Hong Kong. He joined the Department of Electrical and Computer Engineering at UBC in 1989, where he is a Professor and holder of the TELUS Mobility Research Chair in Advanced Telecommunications Engineering. He is also a member of the UBC Centre for Integrated Computer Systems Research. He is a project leader and member of the Board of Directors in the Canadian Institute for Telecommunications Research. His research interests include the areas of architectural and protocol design and performance analysis for computer and telecommunication networks, with applications in satellite, mobile, personal communications, and high speed networks.

Dr. Leung was awarded the APEBC Gold Medal as the head of the graduating class in the Faculty of Applied Science. He is an Associate Editor of the IEEE TRANSACTIONS ON VEHICULAR TECHNOLOGY and an Editor of the IEEE JoURNAL ON SELECTED AREAS IN COMMUNICATIONS-Wireless Communications Series. He is a voting member of the ACM. 